\title{
Dynamic 18F-FDopa PET Imaging for Newly- diagnosed Gliomas: Is a Semi-quantitative Model Sufficient?
}

Timothée ZARAGORI ( $\square$ timothee.zaragori@univ-lorraine.fr)

INSERM U1254: Imagerie Adaptative Diagnostique et Interventionnelle https://orcid.org/0000-00022050-3591

\section{Matthieu Doyen}

INSERM U1254: Imagerie Adaptative Diagnostique et Interventionnelle

\section{Fabien Rech}

CHRU de Nancy: Centre hospitalier regional universitaire de Nancy

\section{Marie Blonski}

CHRU de Nancy: Centre hospitalier regional universitaire de Nancy

\section{Luc Taillandier}

CHRU de Nancy: Centre hospitalier regional universitaire de Nancy

\section{Laetitia Imbert}

CHRU de Nancy: Centre hospitalier regional universitaire de Nancy

\section{Antoine Verger}

CHRU de Nancy: Centre hospitalier regional universitaire de Nancy

\section{Research Article}

Keywords: DOPA, PET, compartmental modelling, dynamic analysis, glioma, IDH mutation

Posted Date: July 26th, 2021

DOI: https://doi.org/10.21203/rs.3.rs-560933/v1

License: (a) (1) This work is licensed under a Creative Commons Attribution 4.0 International License. Read Full License 


\section{Abstract}

Purpose: Even though the semi quantitative dynamic analysis of ${ }^{18}$ F-FDOPA PET effectively and noninvasively predicts isocitrate dehydrogenase (IDH) mutations in newly-diagnosed gliomas, the underlying kinetic model of ${ }^{18} \mathrm{~F}$-FDOPA is complex. Our current study addresses whether a semi quantitative analysis indeed captures all the clinically relevant predictive features of the more sophisticated graphical and compartmental models.

Methods: Thirty-seven tumour time-activity curves from ${ }^{18}$ F-FDOPA PET dynamic acquisitions of newlydiagnosed gliomas were analysed using a semi quantitative model with (Ref SQ) or without reference region (SQ), a graphical Logan model with input function (Logan) or reference region (Ref Logan), and a two-tissue compartmental model validated for ${ }^{18} \mathrm{~F}$-FDOPA PET imaging in gliomas (2TCM). The overall predictive performance of each model for predicting IDH mutations was assessed, by an area under the curve (AUC) comparison of multivariate analyses of all parameters included in the model.

Results: SQ model with an AUC of 0.733 showed comparable performances to other models with AUCs of $0.814,0.693,0.786,0.863$, respectively corresponding to Ref SQ, Logan, Ref Logan and 2 TCM ( $\geq 0.11$ for the pairwise comparisons with other models). SQ time-to-peak parameter had the best diagnostic performance relative to all individual parameters with an accuracy of $75.7 \%$.

Conclusions: The SQ model circumvents the complexities of the ${ }^{18} \mathrm{~F}-\mathrm{FDOPA}$ kinetic model and yields similar performances compared to other models most notably the compartmental model for predicting IDH mutations. This validates the application of the SQ model for the dynamic analysis of ${ }^{18} \mathrm{~F}-\mathrm{FDOPA}$ PET images in routine clinical practice for newly-diagnosed gliomas.

\section{Introduction}

As an adjunct to MRI, amino-acid PET imaging provides additional diagnostic and prognostic information in newly-diagnosed gliomas [1], as has been demonstrated for the most frequently used radiolabelled amino-acids 0-(2-[18F]fluoroethyl)-L-tyrosine $\left({ }^{18} \mathrm{~F}-\mathrm{FET}\right)[2],{ }^{11} \mathrm{C}$-methionine $\left({ }^{11} \mathrm{C}-\mathrm{MET}\right)$ [3], and 6[18F]fluoro-L-DOPA $\left({ }^{18} \mathrm{~F}\right.$-FDOPA) [4]. Dynamic acquisitions are most commonly performed with ${ }^{18} \mathrm{~F}$-FET and ${ }^{18} \mathrm{~F}$-FDOPA whilst dynamic ${ }^{11} \mathrm{C}-\mathrm{MET}$ acquisitions are rare, and limited by the short, $20 \mathrm{~min}$, half-life of this radiolabelled amino acid [5].

Dynamic ${ }^{18} \mathrm{~F}$-FET and ${ }^{18} \mathrm{~F}$-FDOPA acquisitions recently showed encouraging predictive performances for the non-invasive characterisation of IDH mutation status in newly-diagnosed gliomas [2,4], which is considered to be one of the most important molecular parameters in gliomas according to the World Health Organisation's (WHO) 2016 classification [6]. These results were obtained using a semiquantitative model, initially developed from dynamic ${ }^{18} \mathrm{~F}$-FET PET imaging, that can be easily transposed to clinical routine [7]. The model relies on the extraction of two parameters from the time activity curve (TAC) namely time-to-peak (TTP) which is the time between the beginning of the dynamic acquisition and 
the maximal TAC value and the late phase slope of the TAC. This simple model is currently recommended for dynamic ${ }^{18}$ F-FET PET analyses [8]. The same dynamic analysis has also been successfully transposed to ${ }^{18} \mathrm{~F}$-FDOPA dynamic images with the time-to-peak parameter demonstrating a promising $74 \%$ accuracy for predicting the presence of IDH mutations in newly-diagnosed gliomas [4].

The simple dynamic ${ }^{18}$ F-FET PET modelling approach can be applied because, unlike other radiolabelled amino-acids, ${ }^{18} \mathrm{~F}$-FET is not metabolised in cells [9]. Indeed, several studies have shown that ${ }^{18} \mathrm{~F}$-FET kinetics are best modelled using a one-tissue reversible compartmental model with reliable fit and stable kinetic parameters [10-12]. By comparison, the ${ }^{18} \mathrm{~F}$-FDOPA kinetics in gliomas involve a much more complex model which is to date only partially characterised $[13,14]$ because initial models were developed in the context of assessing Parkinson's disease [15]. The complexities of the ${ }^{18} \mathrm{~F}$-FDOPA kinetic model arise from its different peripheral and intracellular metabolic pathways. ${ }^{18} \mathrm{~F}$-FDOPA is metabolised into ${ }^{18} \mathrm{~F}$-labelled metabolites (METS) and ${ }^{18} \mathrm{~F}$-labelled L-3,4-dihydroxy-6-fluoro-3-0-methylphenylalanine (OMFD) in the periphery, with OMFD able to cross the blood-brain barrier bi-directionally. In addition, and unlike ${ }^{18} \mathrm{~F}$-FET, ${ }^{18} \mathrm{~F}$-FDOPA is metabolised inside cells as part of the dopaminergic pathway even though it is unclear whether this holds true for tumour cells. A two-tissue compartmental model only using one input function was nevertheless previously validated for ${ }^{18}$ F-FDOPA imaging in gliomas $[13,14]$. However, to the best of our knowledge, there are currently no studies which validate the use of semi-quantitative dynamic models and their application in routine practice, or which compare this semi-quantitative dynamic model to other dynamic models, such as the validated compartmental ${ }^{18} \mathrm{~F}$-FDOPA PET imaging model, in gliomas $[13,14]$.

The current study therefore aims to validate the use of a semi-quantitative model to investigate ${ }^{18} \mathrm{~F}$ FDOPA dynamic analyses compared to more sophisticated graphical and compartmental models to predict IDH mutations in newly-diagnosed gliomas.

\section{Materials And Methods}

\section{Patients}

We retrospectively selected newly-diagnosed glioma patients for whom ${ }^{18} \mathrm{~F}$-FDopa PET had been performed as part of the initial tumour characterisation, in the Department of Nuclear Medicine at the CHRU of Nancy, between February 2018 and June 2020. The final selection included: (i) patients with a neuropathological diagnosis based on the WHO 2016 classification [6] and with a maximum delay from the time of the ${ }^{18}$ F-FDopa PET of 150 days for diffuse grade II or III gliomas and 60 days for glioblastomas [2,4], (ii) patients with available raw-data for a-posteriori reconstruction and, (iii) patients with a visually abnormal ${ }^{18} \mathrm{~F}$-FDopa uptake, i.e. by excluding isometabolic and photopenic gliomas $[16,17]$. The institutional ethics committee (Comité d'Ethique du CHRU de Nancy - FRANCE) approved the evaluation of retrospective patient data on August 26, 2020. The trial was registered at ClinicalTrials.gov 
(NCT04469244). This research complied with the principles of the Declaration of Helsinki. Informed consent was obtained from all individuals included in the study.

\section{${ }^{18}$ F-FDopa PET Acquisition and Image Reconstruction}

Patients were instructed to fast for at least 4 hours prior to the examination, and were pre-medicated with Carbidopa one hour prior to the examination to increase tracer uptake in the brain [13]. Patients were scanned with a digital Vereos PET/CT camera (Vereos, Philips, Cleveland, Ohio). Immediately after recording CT images (100 kV, $80 \mathrm{mAs})$, a 30-min 3D list-mode PET acquisition was initiated concomitantly to the bolus injection of $2 \mathrm{MBq}$ of ${ }^{18} \mathrm{~F}$-FDOPA per kilogram of body weight. Static PET images were reconstructed from the list mode data acquired 10 to 30 min post-injection $[8,18]$. Dynamic images were reconstructed using two different temporal sampling protocols depending on the dynamic analyses carried out. Where an input function was required, the temporal sampling protocol was similar to what was recommended in the EANM/SNMMI guidelines, i.e. 12 frames of $5 \mathrm{~s}, 6$ frames of $10 \mathrm{~s}, 6$ frames of $30 \mathrm{~s}, 5$ frames of $60 \mathrm{~s}$ and 4 frames of $300 \mathrm{~s}$ [8]. The temporal sampling protocol consisted of $30 \times 60 \mathrm{~s}$ frames for the models not requiring any input function [19].

Static images were reconstructed using the time-of-flight information and a high-resolution protocol with the OSEM 3D algorithm (2 iterations, 10 subsets, a deconvolution of the point spread function (PSF) and $256 \times 256 \times 164$ voxels of $1 \times 1 \times 1 \mathrm{~mm}^{3}$ ) while a protocol with a lower spatial resolution was used for dynamic images to limit the level of noise, i.e. 3 iterations, 15 subsets, without PSF and $128 \times 128 \times 82$ voxels of $2 \times 2 \times 2 \mathrm{~mm}^{3}[20]$.

All images were corrected for attenuation using $\mathrm{CT}$, dead time, random and scattered coincidences during the reconstruction process.

\section{Segmentation}

On static images, the healthy brain uptake was initially measured using a merged volume of interest (VOI) consisting of a crescent-shaped region of interest manually positioned on three consecutive slices of the unaffected hemisphere so as to comprise both white and gray matter, while the tumour VOI was segmented semi-automatically using a threshold of 1.6 healthy brain SUV mean as previously recommended $[19,21,22]$. The arterial input function VOI was subsequently placed into the internal carotid using initial dynamic frames to identify early vascular phases [23].

All volumes of interest were segmented using the LifeX software (lifexsoft.org) [24] and were visually inspected by an experienced physician (A.V.) to ensure the quality of the methods applied. Healthy brain was considered as the reference region due to its non-specific uptake, where required [21]. 


\section{Extraction of time-activity curves}

For dynamic images reconstructed using the protocol with $30 \times 60 \mathrm{~s}$ frames, each dynamic frame was first registered to the associated CT image, in order to correct for any potential patient movement during the acquisition [25]. These transformations, representing the evolution of the patient's movements over time, were interpolated to the time frames of the other protocol for models involving an input function. Indeed, the first frames from images reconstructed with models involving an input function are very short and suffer from noise, which makes the registration very challenging.

Blood and brain time-activity curves (TACs) were extracted by retrieving the SUV $V_{\text {mean }}$ for each frame in their respective VOI. Tumour TACs were computed by retrieving the SUV $V_{\text {mean }}$ for each frame in the volume corresponding to the SUV peak of the tumour VOI on the static image, to represent the most aggressive part of the tumour [19].

\section{Input Function Pre-processing}

Since no arterial blood sampling was performed in this study, an image-derived input function was used for analyses that required one. TACs representing the evolution of the arterial blood activity were obtained from internal carotid VOIs, and were fitted using linear interpolation to the peak followed by a triexponential function after the peak [26]. In the case of ${ }^{18} \mathrm{~F}$-FDOPA, the plasma ${ }^{18} \mathrm{~F}$-FDOPA TAC is obtained after correcting for OMFD as well as other METS created in the peripheral tissues [15]. This plasma ${ }^{18} \mathrm{~F}-$ FDOPA TAC can be obtained from the blood TAC if the haematocrit level and the proportion of each ${ }^{18} \mathrm{~F}$ labelled entity in the respective plasma TACs are known [14]. These values were retrieved from the literature, specifically from Huang et al. [15] who used a haematocrit of $40 \%$ and from Melega et al. [27] who reported the metabolite proportions for patients pre-medicated with $100 \mathrm{mg}$ of Carbidopa which is identical to the pre-medication schedule of our patients. To extrapolate the proportion of metabolites at any time, the measured fractions of plasma radioactivity were fitted using the following equations for the plasma fractions of DOPA, OMFD, and METS respectively:

$$
\begin{gathered}
f_{D O P A}(t)_{p}=1-0.36902735 \times\left(2-e^{-0.03915133 t}-e^{-0.03915214 t}\right) \\
f_{O M F D}(t)_{p}=0.24080881 \times\left(1-e^{-0.03251229 t}\right)+0.43768904 \times\left(1-e^{-0.03251228 t}\right) \\
f_{M E T S}(t)_{p}=1-f_{O M F D}(t)_{p}-f_{D O P A}(t)_{p}
\end{gathered}
$$

where $\mathrm{t}$ is the time in minutes. 


\section{Dynamic models}

The pre-processing steps and input data for each dynamic model are presented in Figure 1.

\section{Semi-quantitative models}

To overcome noise effects, tumour TACs were first fitted using non-linear least square optimisations and a specific tumour vascularisation function (patent WO/2008/053268, entitled "Method and System for Quantification of Tumoral Vascularization") $[4,19,28]$. Semi quantitative (SQ) model parameters, time-topeak (TTP) and slope, were respectively computed as the time from the beginning of the dynamic acquisition to the maximum uptake value and as the slope of the linear regression of the data between the 10th and 30th minute [4]. The reference semi quantitative (Ref SQ) model was conducted as an assessment of other studies where tumor-to-normal brain ratio dynamic values were used to overcome the Carbidopa effect $[4,19,28]$ even though such normalisation was not needed in this study. For this purpose, healthy brain TACs were also fitted similarly to tumour TACs, and TACs ratio $_{\text {representing the }}$ evolution of the ratio between tumour and brain fitted TACs were computed. TTP ratio and slope ratio $_{\text {were }}$ computed from the TACs $s_{\text {ratio }}$ similarly to what was done for tumour TACs.

\section{Graphical models}

Among all graphical models available, the Logan graphical model [29] with the computation of the equilibrium volumes of distribution is particularly suited to ${ }^{18} \mathrm{~F}-\mathrm{FDOPA}$ in gliomas since there is no evidence that ${ }^{18} \mathrm{~F}$-FDOPA is trapped in tumours [13]. The Logan graphical model (Logan) was performed with the slope computed between 15 and 30 min p.i. as previously suggested [13]. The equilibrium volumes of distribution $\mathrm{V}_{\mathrm{ed}}$ and $\mathrm{Int}_{\text {Logan }}$ were computed respectively as the slope and the intercept of the graphical model. This model was also performed using a healthy brain reference region (Ref Logan) as it can be done with the Patlak graphical model in ${ }^{18} \mathrm{~F}$-FDOPA PET imaging in parkinsonian syndromes [30]. Two parameters were also extracted with regression between 15 and 30 min, namely the distribution volume ratio (DVR) and the relative residence time (RRT) computed respectively as the slope and the intercept of the graphical model with the reference region.

\section{Compartmental model}

The model used was a simplified two-tissue compartmental model (2TCM) adapted from the original publication of Huang et al. [15] and already validated for compartmental analysis of ${ }^{18} \mathrm{~F}$-FDOPA imaging in gliomas [14]. Four rate constants $\left(\mathrm{K}_{1}, \mathrm{k}_{2}, \mathrm{k}_{3}, \mathrm{k}_{4}\right)$ as well as the blood volume fraction $\left(\mathrm{V}_{\mathrm{b}}\right)$ were estimated by fitting the two-tissue compartmental model to tumour TACs. The net influx rate constant Ki was computed from the previously estimated four rate constants as 


\section{$K i=\left(K_{1} \times k_{3}\right) /\left(k_{2}+k_{3}\right)$.}

\section{Statistical Analysis}

Categorical variables are expressed as numbers and percentages and continuous variables as medians [first quartile; third quartile] because variables did not follow a normal distribution. Intergroup comparisons were performed with the Chi-squared test for categorical variables and the Mann-Whitney test for continuous variables.

For the overall comparison of the different kinetic models, parameters belonging to the same model were used to construct a multivariate model. This multivariate model was a general linear model with variables selected automatically in a stepwise manner with both forward and backward selection minimising the Akaike Information Criterion. Comparisons of performance of the final models were carried out with the one-sided comparison of superiority pairwise Delong tests [31].

The ability of each individual extracted parameter to predict an IDH mutation was assessed using receiver operating characteristic (ROC) curves from which area under the curve (AUC), sensitivity, specificity and accuracy were computed. The optimal threshold was determined by selecting the point on the curve closest to $(0,1)$. Spearman coefficients were calculated to assess correlations between each extracted parameter of the different models.

$p$ values were adjusted using the Benjamini-Hochberg correction and $p$ values lower than 0.05 were considered significant. Analyses were performed with the R software version 3.6.2 (R Foundation for Statistical Computing, Vienna, Austria).

\section{Results}

\section{Patients}

Thirty-seven patients (median age of 58.3 [20.3; 79.9] years, 16 (43\%) women) were included in this study. According to the WHO 2016 classification of gliomas, 8 gliomas (22\%) were classified as IDH-mutant astroctyomas (among whom 1 anaplastic glioma), 7 (19\%) as IDH-wildtype astrocytomas (among whom 4 anaplastic gliomas), 5 (14\%) as IDH-mutant and 1p/19q co-deleted oligodendrogliomas, 16 (43\%) as IDH-wildtype glioblastomas and 1 (3\%) as IDH-mutant glioblastoma.

\section{Dynamic models}


The SQ model with an AUC of 0.733 showed similar performances to other models with AUCs of 0.814 , $0.693,0.786,0.863$, respectively corresponding to the Ref SQ, Logan, Ref Logan and 2 TCM ( $p \geq 0.11$ for the pairwise comparisons with the other models) (Table 1 and Figure 2). No significant differences were found in the pairwise comparisons of other dynamic models $(p>0.05)$.

Table 1

Results of multivariate analyses for predicting IDH mutations with different dynamic models

\begin{tabular}{|lll|}
\hline Dynamic model & AUC $[95 \% \mathrm{Cl}]$ & $\begin{array}{l}\text { Parameters included in the } \\
\text { multivariate model }\end{array}$ \\
\hline Semi-quantitative & $0.733[0.564 ; 0.901]$ & TTP \\
\hline Reference semi-quantitative & $0.814[0.671 ; 0.956]$ & TTP $_{\text {ratio }}$ \\
\hline Logan & $0.693[0.496 ; 0.889]$ & Int $_{\text {Logan }}$ \\
\hline Reference Logan & $0.786[0.620 ; 0.951]$ & DVR \\
\cline { 3 - 3 } & & RRT \\
\hline tissue compartmental & $0.863[0.734 ; 0.993]$ & $\mathrm{K}_{1}$ \\
& & $\mathrm{k}_{3}$ \\
\cline { 2 - 2 } & & $\mathrm{k}_{4}$ \\
\hline
\end{tabular}

Detailed diagnostic performances of all parameters taken individually and correlations between individual parameters are presented in Table 2 and Figure 3 respectively. The TTP parameter from the SQ model showed the best accuracy $(75.7 \%)$ among all the parameters examined. The other SQ parameter, namely slope, also had a strong diagnostic performance with an accuracy of $73.0 \%$, which is within the range of performances for parameters from the Ref SQ and Ref Logan models (respective accuracies of

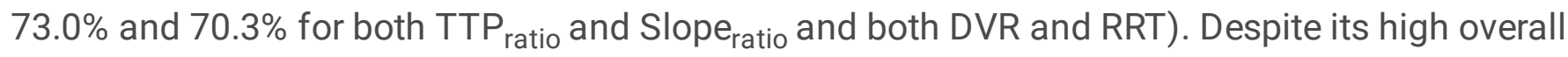
performance, none of the parameters obtained with the 2TCM model were significant for predicting IDH mutations $(p>0.13)$. SQ parameters were highly correlated with Ref SQ Slope $e_{\text {ratio }}$ and the intercepts from

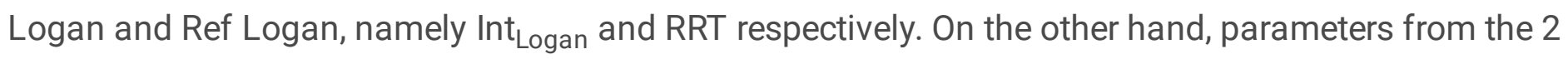
TCM model did not correlate with parameters from other models. Most parameters extracted from this model were highly correlated. Representative examples of gliomas are shown in Figure 4.

Table 2

Results of ROC curve analyses for predicting IDH mutations using individual parameters. 


\begin{tabular}{|c|c|c|c|c|c|c|c|}
\hline Dynamic model & Parameter & $\begin{array}{l}p \\
\text { value }\end{array}$ & AUC & Cut-off & Sensitivity & Specificity & Accuracy \\
\hline \multirow[t]{2}{*}{ Semi-quantitative } & TTP & 0.020 & 0.733 & $\begin{array}{l}18.41 \\
\min \end{array}$ & $57.1 \%$ & $87.0 \%$ & $75.7 \%$ \\
\hline & Slope & 0.020 & 0.730 & $\begin{array}{l}-0.55 \\
\text { SUV.h } h^{-1}\end{array}$ & $64.3 \%$ & $78.3 \%$ & $73.0 \%$ \\
\hline \multirow[t]{2}{*}{$\begin{array}{l}\text { Reference semi- } \\
\text { quantitative }\end{array}$} & TTP $_{\text {ratio }}$ & 0.003 & 0.814 & $\begin{array}{l}5.28 \\
\min \end{array}$ & $78.6 \%$ & $69.6 \%$ & $73.0 \%$ \\
\hline & Slope $_{\text {ratio }}$ & 0.024 & 0.724 & $\begin{array}{l}-0.37 \mathrm{~h}^{-} \\
1\end{array}$ & $78.6 \%$ & $69.6 \%$ & $73.0 \%$ \\
\hline \multirow[t]{2}{*}{ Logan } & $\mathrm{V}_{\text {ed }}$ & 0.156 & 0.643 & 2.91 & $64.3 \%$ & $65.2 \%$ & $64.9 \%$ \\
\hline & Int $_{\text {Logan }}$ & 0.107 & 0.693 & $\begin{array}{l}-25.12 \\
\min \end{array}$ & $57.1 \%$ & $82.6 \%$ & $73.0 \%$ \\
\hline \multirow[t]{2}{*}{ Reference Logan } & DVR & 0.046 & 0.699 & 2.41 & $78.6 \%$ & $65.2 \%$ & $70.3 \%$ \\
\hline & RRT & 0.030 & 0.739 & $\begin{array}{l}0.71 \\
\min \end{array}$ & $78.6 \%$ & $65.2 \%$ & $70.3 \%$ \\
\hline \multirow[t]{6}{*}{$\begin{array}{l}2 \text { tissue } \\
\text { compartmental } \\
\text { model }\end{array}$} & $\mathrm{K}_{1}$ & 0.131 & 0.727 & $\begin{array}{l}0.26 \\
\mathrm{~min}^{-1}\end{array}$ & $71.4 \%$ & $69.6 \%$ & $70.3 \%$ \\
\hline & $\mathrm{k}_{2}$ & 0.464 & 0.609 & $\begin{array}{l}0.40 \\
\min ^{-1}\end{array}$ & $64.3 \%$ & $52.2 \%$ & $56.8 \%$ \\
\hline & $\mathrm{k}_{3}$ & 0.888 & 0.516 & $\begin{array}{l}0.18 \\
\min ^{-1}\end{array}$ & $57.1 \%$ & $56.5 \%$ & $56.8 \%$ \\
\hline & $\mathrm{k}_{4}$ & 0.257 & 0.671 & $\begin{array}{l}0.03 \\
\min ^{-1}\end{array}$ & $71.4 \%$ & $65.2 \%$ & $67.6 \%$ \\
\hline & $\mathrm{Ki}$ & 0.464 & 0.590 & $\begin{array}{l}0.07 \\
\min ^{-1}\end{array}$ & $64.3 \%$ & $56.5 \%$ & $59.5 \%$ \\
\hline & $\mathrm{V}_{\mathrm{b}}$ & 0.464 & 0.587 & $3.86 \%$ & $71.4 \%$ & $56.5 \%$ & $62.2 \%$ \\
\hline
\end{tabular}

Bold $p$ values are significant Mann Whitney tests for the comparison of IDH wildtype gliomas and IDH mutant gliomas.

\section{Discussion}

The present study compared different models of ${ }^{18} \mathrm{~F}$-FDOPA kinetics to assess whether the SQ model, which is currently recommended for use in clinical routine with amino-acid PET, is specifically suited to a ${ }^{18}$ F-FDOPA PET dynamic analysis [8]. This comparison was performed on a newly-diagnosed gliomas for 
which the dynamic analysis of amino-acid PET had previously been shown to successfully predict IDH mutation status $[2,4,19]$. The SQ model was found to have a similar performance than the other models including the 2 TCM model, validated for dynamic ${ }^{18}$ F-FDOPA PET imaging of gliomas $[13,14]$ (Table 1, Figure 2). Our current study therefore validates the application of the SQ model, to predict IDH mutations by ${ }^{18} \mathrm{~F}$-FDOPA PET imaging, in clinical routine practice for newly-diagnosed gliomas.

The SQ model has the advantage of being easily integrated into clinical routine with no need for heavy processing, a reference region or an input function. The input function is the basis of the compartmental analysis currently considered as the gold standard. However, an input function is difficult to obtain in clinical routine because it either requires blood sampling or, if extracted directly from images, heavy postprocessing and many approximations (see Figure 1) limiting its use in clinical practice.

There has been renewed interest in dynamic PET acquisitions due to significant technological advances in the field, such as digital signal [32] and/or longer field of view of the PET devices. These improvements make dynamic acquisitions accessible to clinical routine practice [33]. A simple dynamic model is thus preferable to harmonise protocols across centres and to promote a wider acceptance in the scientific medical community, both among nuclear physicians but also clinicians requiring access to easily understandable and interpretable data.

From a diagnostic performance standpoint our study validates the high diagnostic performances of dynamic ${ }^{18}$ F-FDOPA PET imaging using the SQ model to predict IDH mutations, with the TTP analysis yielding an accuracy of $75.7 \%$. Such performances are within the range of previously published studies reporting accuracies of $74 \%$ for the TTP alone [4] and $75 \%$ in association with other specific radiomics features of ${ }^{18} \mathrm{~F}$-FDOPA PET imaging [19]. It should nevertheless be noted that these latter two studies used a reference region for the SQ model to account for the population heterogeneity as regards Carbidopa pre-medication $[4,19,28]$. In the current study, we report a similar overall performance for the Ref SQ model and the SQ model (Ref SQ AUC: 0.814 [0.671; 0.956], $p=0.21$ for the pairwise test with SQ model), this validates results obtained in our previous dynamic ${ }^{18}$ F-FDOPA PET studies $[4,19,28]$. Even though no significant differences were observed between the different models studied, the 2 TCM model provided better performances (AUC: 0.863 [0.734; 0.993], $p=0.11$ for the pairwise test with SQ model) with parameters that reflect different kinetic information compared to parameters from the other models (Figure 3). However, as explained above, adapting the 2 TCM model to clinical routine would be very challenging and would be more difficult to harmonise between centres.

Among the radiolabelled amino-acids recommended for the assessment of gliomas, ${ }^{18} \mathrm{~F}-\mathrm{FET}$ and ${ }^{18} \mathrm{~F}$ FDOPA have been demonstrated to have similar semi-quantitative static parameters [34] but different kinetic parameters for tumour grading using compartmental modelling [23]. When applying the WHO 2016 classification of gliomas, dynamic parameters for both radiotracers extracted from SQ or Ref SQ models, in the literature, showed similar performances for predicting IDH mutations. An accuracy of $72 \%$ was reported for the ${ }^{18}$ F-FET TTP [2] while an accuracy of $74 \%$ was described for the ${ }^{18}$ F-FDOPA TTP ratio [4]. These results are also confirmed in the Lohmann et al. radiomics study which reports a model 
accuracy of 70\% [35]. In this study, dynamic parameters were nevertheless particularly pertinent for determining the diagnostic performance. Since the underlying kinetic model of ${ }^{18} \mathrm{~F}$-FDOPA is much more complex than ${ }^{18} \mathrm{~F}$-FET, there was no evidence that a simple model such as SQ model would suffice. Our current study showed that the SQ model is appropriate for interpreting ${ }^{18} \mathrm{~F}$-FDOPA kinetics and that it might be considered in the future guidelines as is currently the case for ${ }^{18} \mathrm{~F}$-FET imaging [8].

The main limitations of our study are related to the assumptions which underpin how the input function was determined. Since no blood sampling was performed for any of our patients, we extrapolated the input function from an image-derived blood TAC that was converted to ${ }^{18} \mathrm{~F}$-FDOPA plasma TAC using a previously published method [14]. This method is based on haematocrit data and the proportion of individual metabolites previously reported in the literature $[15,27]$. Since this assumption is not adapted to each patient it can negatively affect results from the model that are dependent of this input function. Moreover, our study used a digital PET device with high count sensitivities which yields a better quality of dynamic images. The values of the different parameters may therefore not be directly comparable to those extracted from noisier dynamic images, captured with older PET devices. This may significantly affect the fitting process that is used for each dynamic model and thus affect the stability and the ability to directly extrapolate our results to other PET devices.

\section{Conclusion}

Despite the complexity of the ${ }^{18} \mathrm{~F}$-FDOPA kinetics model, our study validates the application of a simple semi-quantitative model, which is currently recommended for amino-acid PET imaging in clinical routine practice, for the prediction of IDH mutations with dynamic ${ }^{18} \mathrm{~F}$-FDOPA PET images. While dynamic parameters play an important role in the interpretation of radiolabelled amino-acid PET imaging in gliomas, our current study shows that the application of this easily transposable method can be extended for ${ }^{18}$ F-FDOPA PET imaging to the non-invasive characterisation of newly-diagnosed gliomas.

\section{Declarations}

\section{Ethics approval and consent to participate:}

All procedures in studies involving human participants were performed in accordance with the ethical standards of the institutional and/or national research committee and with the latest amendments of the 1964 Helsinki declaration or comparable ethical standards. Written informed consent was obtained from all individual participants included in the study. The institutional ethics committee (Comité d'Ethique du CHRU de Nancy - FRANCE) approved the evaluation of retrospective patient data on August 26, 2020. The trial was registered at ClinicalTrials.gov (NCT04469244).

\section{Consent for publication:}




\section{Availability of data and materials:}

All data generated or analysed during this study are included in this published article and its supplementary information files.

\section{Competing interests:}

All authors declare that they have no competing interests.

\section{Funding:}

None

\section{Author's contributions:}

All authors participated either in the: 1) study conception and design or analysis and interpretation of the data, or both (TZ, MD, AV); 2) drafting of the manuscript or revising it critically for important intellectual content (TZ, MD, FR, MB, LT, LI, AV); 3) final approval of the submitted manuscript (FR, MB, LT, LI, AV).

\section{Acknowledgements:}

The authors thank Petra Neufing for critical review of the manuscript.

\section{References}

1. Albert NL, Weller M, Suchorska B, Galldiks N, Soffietti R, Kim MM, et al. Response Assessment in Neuro-Oncology working group and European Association for Neuro-Oncology recommendations for the clinical use of PET imaging in gliomas. Neuro-Oncol. 2016;18:1199-208.

2. Verger A, Stoffels G, Bauer EK, Lohmann P, Blau T, Fink GR, et al. Static and dynamic 18F-FET PET for the characterization of gliomas defined by IDH and 1p/19q status. Eur J Nucl Med Mol Imaging. 2018;45:443-51.

3. Lopci E, Riva M, Olivari L, Raneri F, Soffietti R, Piccardo A, et al. Prognostic value of molecular and imaging biomarkers in patients with supratentorial glioma. Eur J Nucl Med Mol Imaging. 2017;44:1155-64.

4. Ginet M, Zaragori T, Marie P-Y, Roch V, Gauchotte G, Rech F, et al. Integration of dynamic parameters in the analysis of 18F-FDopa PET imaging improves the prediction of molecular features of gliomas. 
Eur J Nucl Med Mol Imaging. 2020;47:1381-90.

5. Nomura Y, Asano Y, Shinoda J, Yano H, Ikegame Y, Kawasaki T, et al. Characteristics of time-activity curves obtained from dynamic 11C-methionine PET in common primary brain tumors. J Neurooncol. 2018;138:649-58.

6. Louis DN, Perry A, Reifenberger G, von Deimling A, Figarella-Branger D, Cavenee WK, et al. The 2016 World Health Organization Classification of Tumors of the Central Nervous System: a summary. Acta Neuropathol (Berl). 2016;131:803-20.

7. Pöpperl G, Kreth FW, Herms J, Koch W, Mehrkens JH, Gildehaus FJ, et al. Analysis of 18F-FET PET for grading of recurrent gliomas: is evaluation of uptake kinetics superior to standard methods? J Nucl Med. 2006;47:393-403.

8. Law I, Albert NL, Arbizu J, Boellaard R, Drzezga A, Galldiks N, et al. Joint EANM/EANO/RANO practice guidelines/SNMMI procedure standards for imaging of gliomas using PET with radiolabelled amino acids and [18F]FDG: version 1.0. Eur J Nucl Med Mol Imaging. 2019;46:540-57.

9. Wester HJ, Herz M, Weber W, Heiss P, Senekowitsch-Schmidtke R, Schwaiger M, et al. Synthesis and radiopharmacology of O-(2-[18F]fluoroethyl)-L-tyrosine for tumor imaging. J Nucl Med. 1999;40:20512.

10. Debus C, Afshar-Oromieh A, Floca R, Ingrisch M, Knoll M, Debus J, et al. Feasibility and robustness of dynamic 18F-FET PET based tracer kinetic models applied to patients with recurrent high-grade glioma prior to carbon ion irradiation. Sci Rep. 2018;8:14760.

11. Bolcaen J, Lybaert K, Moerman L, Descamps B, Deblaere K, Boterberg T, et al. Kinetic Modeling and Graphical Analysis of 18F-Fluoromethylcholine (FCho), 18F-Fluoroethyltyrosine (FET) and 18FFluorodeoxyglucose (FDG) PET for the Fiscrimination between High-Grade Glioma and Radiation Necrosis in Rats. PLOS ONE. 2016;11:e0161845.

12. Thiele F, Ehmer J, Piroth MD, Eble MJ, Coenen HH, Kaiser H-J, et al. The quantification of dynamic FET PET imaging and correlation with the clinical outcome in patients with glioblastoma. Phys Med Biol. 2009;54:5525-39.

13. Schiepers C, Chen W, Cloughesy T, Dahlbom M, Huang S-C. 18F-FDOPA Kinetics in Brain Tumors. J Nucl Med. 2007:48:1651-61.

14. Wardak M, Schiepers C, Cloughesy TF, Dahlbom M, Phelps ME, Huang S-C. 18F-FLT and 18F-FDOPA PET kinetics in recurrent brain tumors. Eur J Nucl Med Mol Imaging. 2014;41:1199-209.

15. Huang S-C, Yu D-C, Barrio JR, Grafton S, Melega WP, Hoffman JM, et al. Kinetics and Modeling of I-6-[ 18 F]Fluoro-DOPA in Human Positron Emission Tomographic Studies. J Cereb Blood Flow Metab. 1991;11:898-913.

16. Galldiks N, Unterrainer M, Judov N, Stoffels G, Rapp M, Lohmann P, et al. Photopenic defects on O-(2[18F]-fluoroethyl)-L-tyrosine PET: clinical relevance in glioma patients. Neuro-Oncol. 2019;21:13318.

17. Zaragori T, Castello A, Guedj E, Girard A, Galldiks N, Albert NL, et al. Photopenic Defects in Gliomas With Amino-Acid PET and Relative Prognostic Value: A Multicentric 11C-Methionine and 18F-FDOPA 
PET Experience. Clin Nucl Med. 2021;46:e36-7.

18. Janvier L, Olivier P, Blonski M, Morel O, Vignaud J-M, Karcher G, et al. Correlation of SUV-Derived Indices With Tumoral Aggressiveness of Gliomas in Static 18F-FDOPA PET: Use in Clinical Practice. Clin Nucl Med. 2015;40:e429-35.

19. Zaragori T, Oster J, Roch V, Hossu G, Chawki MB, Grignon R, et al. ${ }^{18}$ F-FDOPA PET for the noninvasive prediction of glioma molecular parameters: a radiomics study. J Nucl Med. 2021;jnumed.120.261545.

20. Salvadori J, Imbert L, Perrin M, Karcher G, Lamiral Z, Marie P-Y, et al. Head-to-head comparison of image quality between brain 18F-FDG images recorded with a fully digital versus a last-generation analog PET camera. EJNMMI Res. 2019;9:61.

21. Unterrainer M, Vettermann F, Brendel M, Holzgreve A, Lifschitz M, Zähringer M, et al. Towards standardization of 18F-FET PET imaging: do we need a consistent method of background activity assessment? EJNMMI Res. 2017;7:48.

22. Cicone F, Carideo L, Minniti G, Scopinaro F. The mean striatal 18F-DOPA uptake is not a reliable cutoff threshold for biological tumour volume definition of glioma. Eur J Nucl Med Mol Imaging. 2019;46:1051-3.

23. Kratochwil C, Combs SE, Leotta K, Afshar-Oromieh A, Rieken S, Debus J, et al. Intra-individual comparison of 18F-FET and 18F-DOPA in PET imaging of recurrent brain tumors. Neuro-Oncol. 2014;16:434-40.

24. Nioche C, Orlhac F, Boughdad S, Reuzé S, Goya-Outi J, Robert C, et al. LIFEx: A Freeware for Radiomic Feature Calculation in Multimodality Imaging to Accelerate Advances in the Characterization of Tumor Heterogeneity. Cancer Res. 2018;78:4786-9.

25. Ye H, Wong K-P, Wardak M, Dahlbom M, Kepe V, Barrio JR, et al. Automated Movement Correction for Dynamic PET/CT Images: Evaluation with Phantom and Patient Data. PLoS ONE. 2014;9:e103745.

26. Zanotti-Fregonara P, Hirvonen J, Lyoo CH, Zoghbi SS, Rallis-Frutos D, Huestis MA, et al. PopulationBased Input Function Modeling for [18F]FMPEP-d2, an Inverse Agonist Radioligand for Cannabinoid CB1 Receptors: Validation in Clinical Studies. PLoS ONE. 2013;8:e60231.

27. Melega WP, Grafton ST, Huang S-C, Satyamurthy N, Phelps ME, Barrio JR. L-6-[ ${ }^{18}$ F]Fluoro-DOPA Metabolism in Monkeys and Humans: Biochemical Parameters for the Formulation of Tracer Kinetic Models with Positron Emission Tomography. J Cereb Blood Flow Metab. 1991;11:890-7.

28. Zaragori T, Ginet M, Marie P-Y, Roch V, Grignon R, Gauchotte G, et al. Use of static and dynamic [ 18 F]-F-DOPA PET parameters for detecting patients with glioma recurrence or progression. EJNMMI Res. 2020;10:1-10.

29. Logan J. Graphical analysis of PET data applied to reversible and irreversible tracers. Nucl Med Biol. 2000;27:661-70.

30. Morbelli S, Esposito G, Arbizu J, Barthel H, Boellaard R, Bohnen NI, et al. EANM practice guideline/SNMMI procedure standard for dopaminergic imaging in Parkinsonian syndromes 1.0. Eur J Nucl Med Mol Imaging. 2020;47:1885-912. 
31. DeLong ER, DeLong DM, Clarke-Pearson DL. Comparing the Areas under Two or More Correlated Receiver Operating Characteristic Curves: A Nonparametric Approach. Biometrics. 1988;44:837.

32. Salvadori J, Odille F, Verger A, Olivier P, Karcher G, Marie P-Y, et al. Head-to-head comparison between digital and analog PET of human and phantom images when optimized for maximizing the signalto-noise ratio from small lesions. EJNMMI Phys. 2020;7:11.

33. Dimitrakopoulou-Strauss A, Pan L, Sachpekidis C. Kinetic modeling and parametric imaging with dynamic PET for oncological applications: general considerations, current clinical applications, and future perspectives. Eur J Nucl Med Mol Imaging. 2021;48:21-39

34. Lapa C, Linsenmann T, Monoranu CM, Samnick S, Buck AK, Bluemel C, et al. Comparison of the amino acid tracers 18F-FET and 18F-DOPA in high-grade glioma patients. J Nucl Med. 2014;55:1611-6.

35. Lohmann P, Lerche C, Bauer EK, Steger J, Stoffels G, Blau T, et al. Predicting IDH genotype in gliomas using FET PET radiomics. Sci Rep. 2018;8:13328.

\section{Figures}




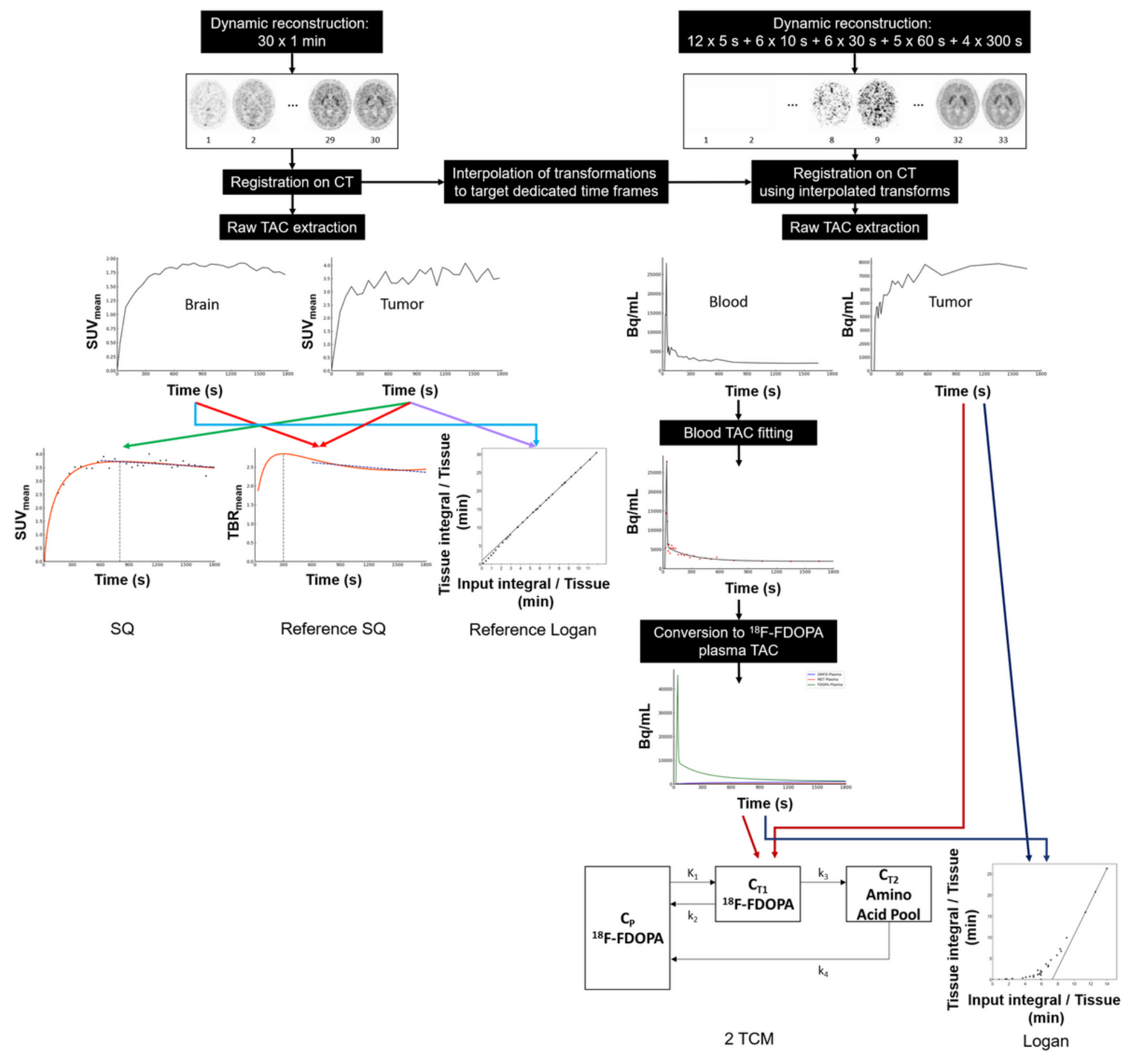

Figure 1

Workflow showing the pre-processing steps required to perform dynamic analyses for all of the dynamic models used in this study: semi-quantitative model (SQ), reference semi-quantitative model (Reference SQ), Logan model with input function (Logan), Logan model with reference region (Reference Logan) and two-tissue compartmental model (2 TCM). TBR: tumor-to-brain ratio. 


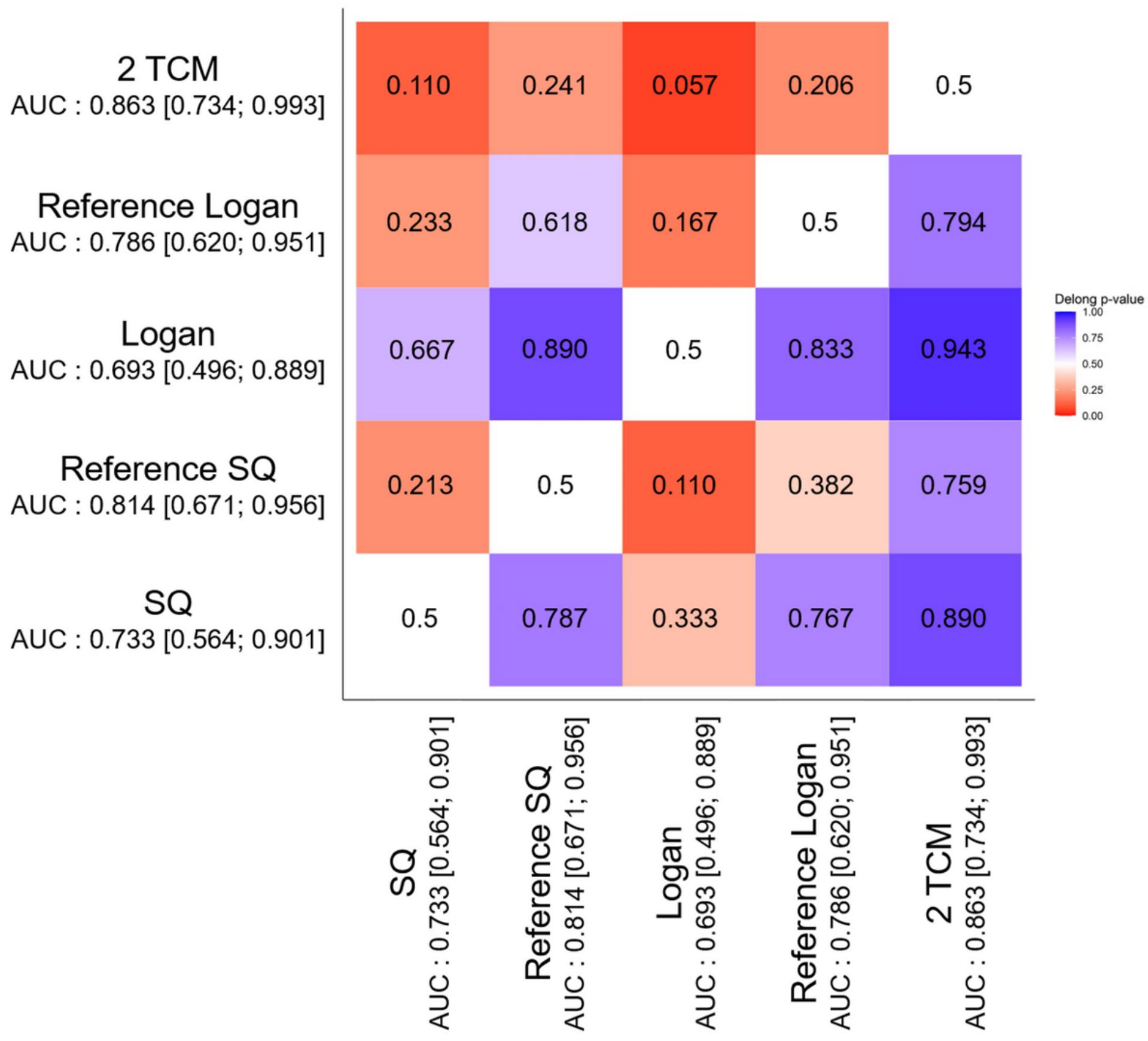

Figure 2

Heatmap of $\mathrm{p}$ values for one-sided comparison of superiority pairwise Delong tests of AUC multivariate analyses performed for each dynamic model. AUC: area-under-the-curve; SQ: Semi-quantitative model; Reference SQ: Reference semi-quantitative model; 2 TCM: two-tissue compartmental model. 


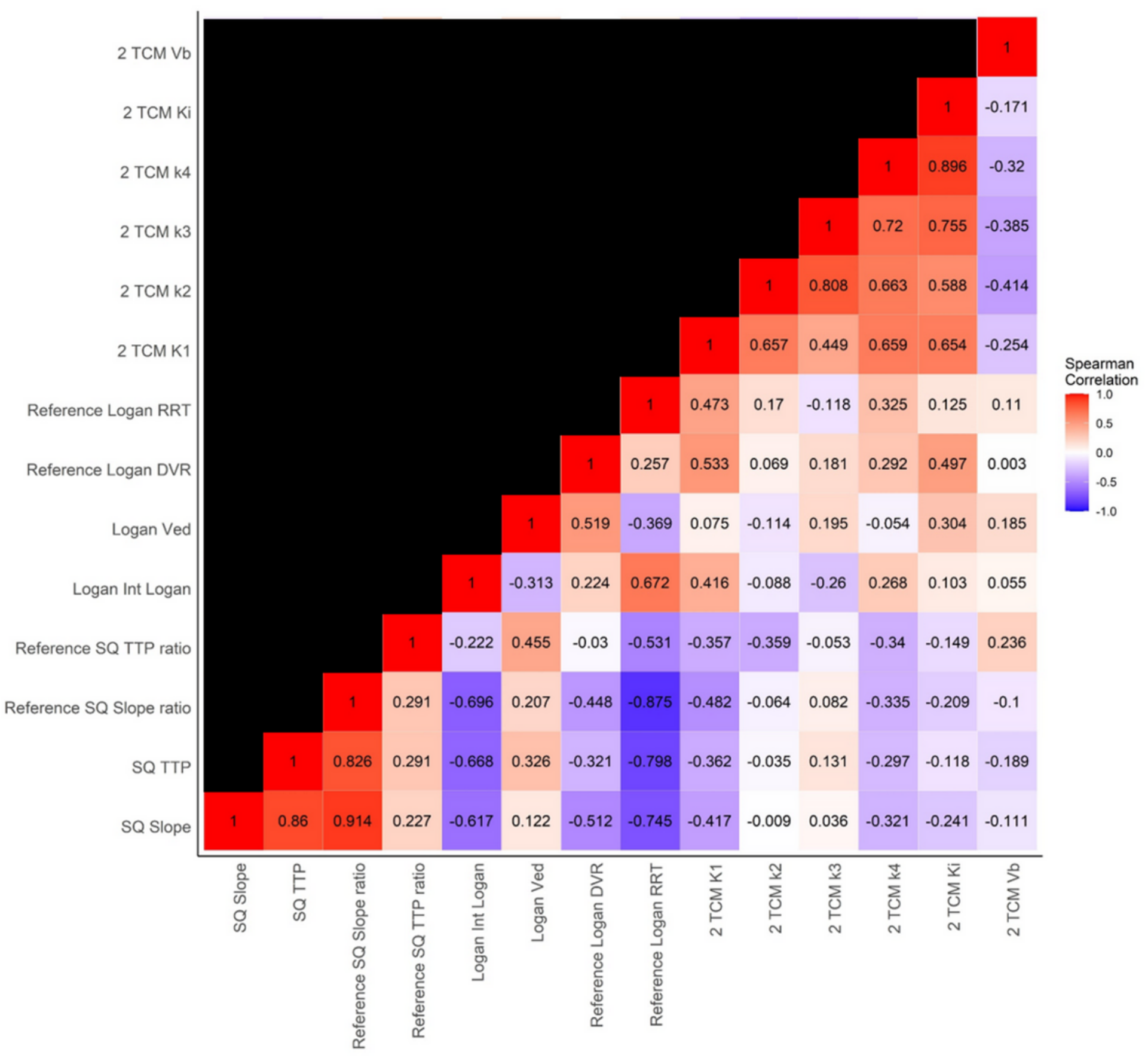

\section{Figure 3}

Heatmap of Spearman correlation coefficients for individual parameters of each of the models. 


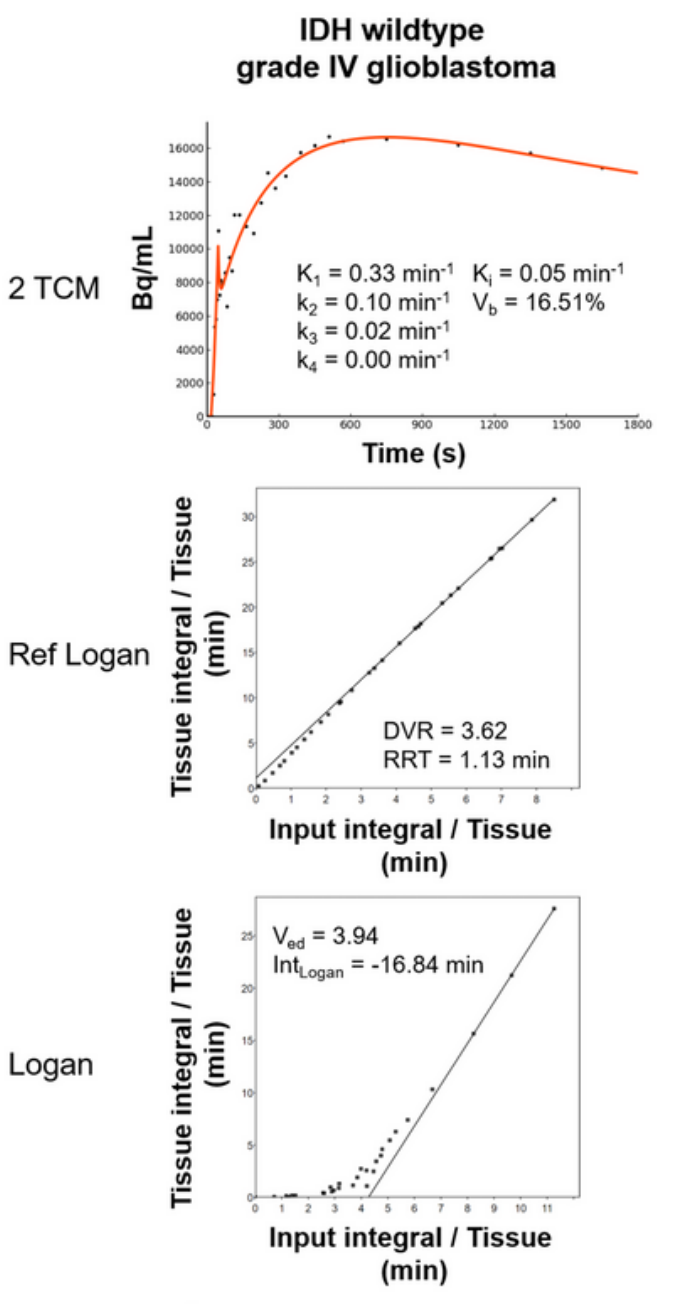

\section{IDH mutant 1p/19q co-deleted grade II oligodendroglioma}
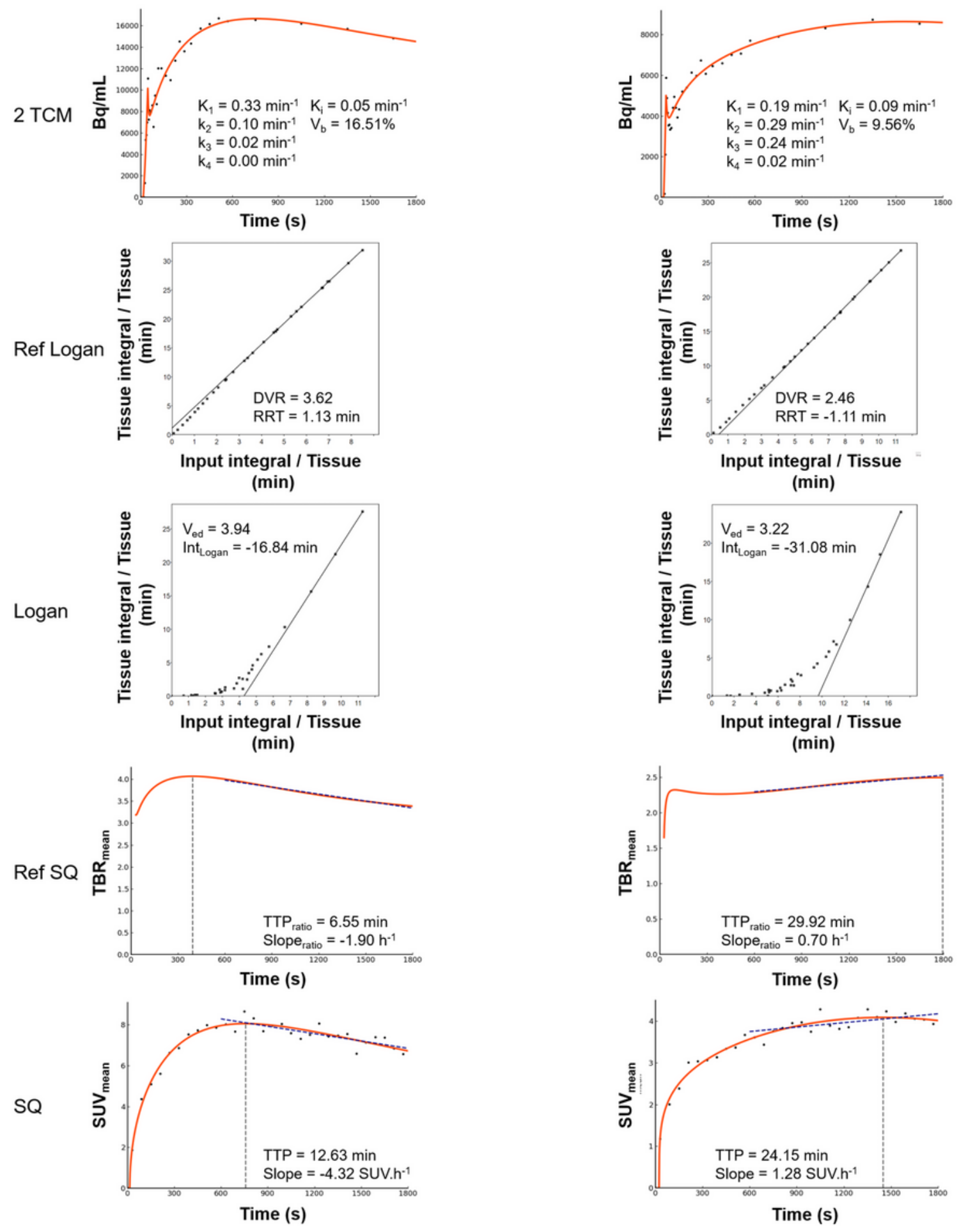

\section{Figure 4}

A representative IDH wildtype, grade IV glioblastoma and an IDH mutant and 1p/19q co-deleted grade II oligodendroglioma. Fitted results and parameters extracted from all dynamic models used in this study are displayed for each of the two patients: semi-quantitative model (SQ), reference semi-quantitative model (Reference SQ), Logan model with input function (Logan), Logan model with reference region (Reference Logan) and two-tissue compartmental model (2 TCM). 


\section{Supplementary Files}

This is a list of supplementary files associated with this preprint. Click to download.

- Supplementaldata.xlsx 